\title{
Local controllability of a magnetized Purcell's swimmer
}

\author{
Clément Moreau*
}

\begin{abstract}
This paper focuses on the control theory aspects of the dynamics of a magnetized micro-swimmer robot model made of three rigid links. Under generic assumptions on the parameters, we show that the control system which describes the swimmer dynamics is locally controllable in small time around its equilibrium position (the straight line), but with bounded controls that do not go to zero as the target state gets closer to the initial state. This result is relevant for useful applications in the micro-swimming field, and provides better understanding of this type of two-control systems.
\end{abstract}

Index Terms-Control applications, robotics, micro-swimming.

\section{INTRODUCTION}

$\mathbf{M}$ ICRO-SWIMMING robots offer potential high-impact applications in the biomedical field, such as targeted drug delivery or non-invasive surgery. For that reason, the interest in building such robots has been growing in the past years. The shapes and propulsion techniques of these new robots could be inspired by biology, since micro-organisms such as sperm cells or bacterias developed efficient ways to move through a surrounding fluid (see [1]). One promising technique consists of using an external magnetic field to drive a magnetized swimmer (see [2], [3], [4]).

In this paper, we focus on this type of propulsion, applied on a simple model of micro-swimmer consisting of three magnetized segments linked by elastic joints. Such models, with different numbers of segments, have been studied for instance in [5] and [6], in which the authors show that sinusoidal magnetic fields allow the swimmer to move forward in a prescribed direction. The 3-link articulated swimmer was introduced by Purcell in a founding talk about microswimming [7].

At the microscopic scale, the Reynolds number is typically very small (around $10^{-6}$ ), which means that the intensity of inertial forces is negligible compared to those of viscous ones. Therefore, we can assume that the fluid is governed by the Stokes equations. We model the hydrodynamic interaction between the swimmer and the fluid by the local drag approximation of Resistive Force Theory introduced in [8].

We state a local controllability result for the 3-link swimmer. Under generic conditions on the links magnetizations, we show that it is controllable around its equilibrium position (a straight line), but with controls that cannot be made arbitrarily small. This is due to the fact that the parallel component of the magnetic field cannot act on the swimmer when all its links are aligned. The proof gives an explicit bound on the controls to achieve small-time local controllability (STLC).

\footnotetext{
*Université Côte d'Azur, Inria, Team McTAO, France clement.moreaudinria.fr
}

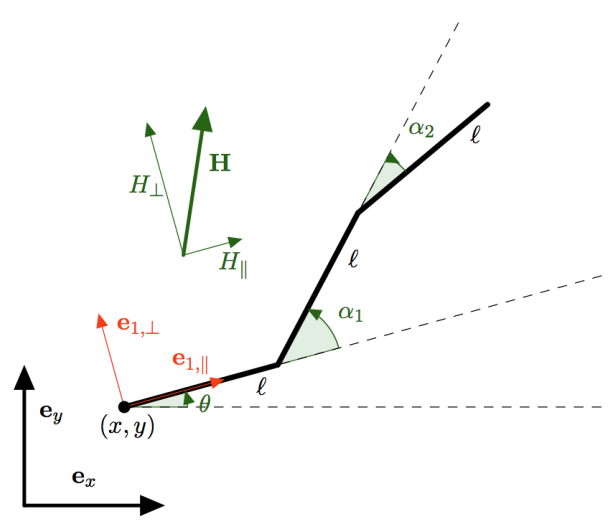

Fig. 1. 3-link microswimmer model

In [9], the authors show a similar local controllability result for the 2-link model around its straight position. The method that we present here to obtain our controllability result allows to improve this result with a stronger form of local controllability. In addition to its interest for applications and experiments, our result raises potential new STLC conditions for a particular class of control systems.

The paper is organized as follows. In Section II, we describe the micro-swimmer model and derive the dynamics equations. In Section III, we recall definitions and results regarding smalltime local controllability (STLC), state our main result, and extend it to the 2-link swimmer model. Section IV is dedicated to a discussion about the results as well as some numerical simulations.

\section{MICRO-SWIMMER MODEL}

\section{A. Formulation of the problem}

We mostly follow the notations and model used in [10], [9] and [11]. We focus on a micro-swimmer consisting of 3 rigid magnetized segments - see Figure 1 - connected by two torsional springs with stiffness $\kappa$, subject to a uniform in space, time-varying magnetic field $\mathbf{H}$. The 3 segments, called $S_{1}, S_{2}$ and $S_{3}$, have same length $\ell$, same hydrodynamic drag coefficients $\xi$ and $\eta$, and respective magnetic moments $M_{1}$, $M_{2}$ and $M_{3}$. The swimmer can move in the 2d-plane defined by the vectors $\mathbf{e}_{x}$ and $\mathbf{e}_{y}$. Let $\mathbf{e}_{z}=\mathbf{e}_{x} \times \mathbf{e}_{y}$. Let $\mathbf{x}=(x, y)$ be the coordinates of the end of $S_{1}, \theta$ the angle between $(O x)$ and $S_{1}$, and $\alpha_{1}$ and $\alpha_{2}$ the angles between $S_{1}$ and $S_{2}$ and between $S_{2}$ and $S_{3}$. The swimmer is then completely described by the five state variables $\left(x, y, \theta, \alpha_{1}, \alpha_{2}\right)$ : the pair $(x, y)$ represents the position of the swimmer, $\theta$ its orientation and the pair $\left(\alpha_{1}, \alpha_{2}\right)$ its shape. Let us also define the moving frames associated to $S_{i}$ for $i=1,2,3$ as $\left(\mathbf{e}_{i, \|}, \mathbf{e}_{i, \perp}\right)$. 
The magnetic field $\mathbf{H}(t)$ induces a torque in each of the segments. As it moves, the swimmer experiences hydrodynamic drag, as well as elastic restoring torques at the joints between the segments.

1) Elasticity: The torsional springs which connect the swimmer segments exert a torque $\mathbf{T}^{\mathrm{el}}$ proportional to the shape angles $\alpha_{1}$ and $\alpha_{2}$. Hence the torque $\mathbf{T}_{2}^{\mathrm{el}}$ exerted on $S_{2}$ is given by $\mathbf{T}_{2}^{\mathrm{el}}=\kappa \alpha_{1} \mathbf{e}_{z}$ and the torque $\mathbf{T}_{3}^{\mathrm{el}}$ exerted on $S_{3}$ is given by $\mathbf{T}_{3}^{\mathrm{el}}=\kappa \alpha_{2} \mathbf{e}_{z}$.

The springs tend to get the swimmer back to a straight shape, in which $S_{1}, S_{2}$ and $S_{3}$ are aligned.

2) Hydrodynamics: The fluid surrounding the swimmer exerts a hydrodynamic drag on it. We use the Resistive Force Theory [8] to model this interaction, i.e. the drag force per unit of length is proportional to the velocity and to the hydrodynamics coefficients $\xi$ and $\eta$. For $s \in[0, \ell]$, let $\mathbf{x}_{s}$ be the point of arclength $s$ on one of the segments $S_{i}$. Its velocity $\mathbf{u}_{i}\left(\mathbf{x}_{s}\right)$ is given in the moving frame $\left(\mathbf{e}_{i, \|}, \mathbf{e}_{i, \perp}\right)$ by $\mathbf{u}_{i}\left(\mathbf{x}_{s}\right)=u_{i, \|} \mathbf{e}_{i, \|}+u_{i, \perp} \mathbf{e}_{i, \perp}$. The drag force exerted on this point is then given by

$$
\mathbf{f}_{i}\left(\mathbf{x}_{s}\right)=-\xi u_{i, \|} \mathbf{e}_{i, \|}-\eta u_{i, \perp} \mathbf{e}_{i, \perp} .
$$

Integrating over $S_{i}$ to obtain the total force $\mathbf{F}_{i}^{h}$ exerted on $S_{i}$ :

$$
\mathbf{F}_{i}^{h}=\int_{S_{i}} \mathbf{f}_{i}\left(\mathbf{x}_{s}\right) d \mathbf{x}_{s} .
$$

Moreover, given a point $\mathrm{x}_{0}$, the drag torque for $S_{i}$ with respect to $\mathrm{x}_{0}$ takes the form

$$
\mathbf{T}_{i, \mathbf{x}_{0}}^{h}=\int_{S_{i}}\left(\mathbf{x}_{s}-\mathbf{x}_{0}\right) \times \mathbf{f}_{i}\left(\mathbf{x}_{s}\right) d \mathbf{x}_{s}
$$

Hydrodynamic drag effects are resistant: without a magnetic field, the swimmer tends to immobilize at its equilibrium straight shape.

3) Magnetism: The magnetic field exerts a torque $\mathbf{T}_{i}^{m}$ on $S_{i}$ which is proportional to its magnetization coefficient $M_{i}$ : $\mathbf{T}_{i}^{m}=M_{i} \mathbf{e}_{i, \|} \times \mathbf{H}$.

4) Dynamics equations: The swimmer is considered sufficiently small to be at low Reynolds number regime, and, as a result, inertia may be neglected [7]. Newton's second law says that the total force applied to $\left\{S_{1}+S_{2}+S_{3}\right\}$ is zero, and so is the total torque with respect to $\mathbf{x}$. Same holds for the subsystems $\left\{S_{2}+S_{3}\right\}$ and $\left\{S_{3}\right\}$, with torques computed with respect to the end of, respectively, $S_{2}$ and $S_{3}$. This leads to the following system of equations:

$$
\left\{\begin{array}{llll}
\mathbf{F}_{1}^{h}+\mathbf{F}_{2}^{h}+\mathbf{F}_{3}^{h} & & =0, \\
\mathbf{T}_{1, \mathbf{x}}^{h}+\mathbf{T}_{2, \mathbf{x}}^{h}+\mathbf{T}_{3, \mathbf{x}}^{h} & +\mathbf{T}_{1}^{m}+\mathbf{T}_{2}^{m}+\mathbf{T}_{3}^{m} & = & 0, \\
\mathbf{T}_{2, \mathbf{x}_{2}}^{h}+\mathbf{T}_{3, \mathbf{x}_{2}}^{h} & +\mathbf{T}_{2}^{m}+\mathbf{T}_{3}^{m} & +\underbrace{\mathbf{T}_{2}^{\mathrm{el}}}_{\text {magnetic terms }}+\underbrace{\mathbf{T}_{3}^{\mathrm{el}}}_{\text {elastic terms }}= & =0, \\
\underbrace{\mathbf{T}_{3, \mathbf{x}_{3}}^{h}}_{\text {hydrodynamic terms }}+\underbrace{m}_{3} & =0 .
\end{array}\right.
$$

This system gives five scalar equations by projecting the first line on $(O x)$ and $(O y)$ and the last three on $(O z)$. We project the uniform time-varying magnetic field $\mathbf{H}(t)$ in the moving frame associated to $S_{1}: \mathbf{H}(t)=H_{\|} \mathbf{e}_{1, \|}+H_{\perp} \mathbf{e}_{1, \perp}$, seeing them as control functions. After computing the different contributions with respect to the parameters, the system can be written as an implicit differential system

$$
M\left(\alpha_{1}, \alpha_{2}\right) R_{-\theta} \dot{Z}=Y,
$$

with $Z=\left(\begin{array}{lllll}x & y & \theta & \alpha_{1} & \alpha_{2}\end{array}\right)^{T}$,

$$
R_{\theta}=\left(\begin{array}{cc|c}
\cos \theta & \sin \theta & 0 \\
-\sin \theta & \cos \theta & 0 \\
\hline 0 & 0 & I_{3}
\end{array}\right),
$$

$$
Y=\left(\begin{array}{c}
0 \\
0 \\
H_{\|}\left(M_{2} \sin \alpha_{1}+M_{3} \sin \left(\alpha_{1}+\alpha_{2}\right)\right) \\
-H_{\perp}\left(M_{1}+M_{2} \cos \alpha_{1}+M_{3} \cos \left(\alpha_{1}+\alpha_{2}\right)\right) \\
-\kappa \alpha_{1}+H_{\|}\left(M_{2} \sin \alpha_{1}+M_{3} \sin \left(\alpha_{1}+\alpha_{2}\right)\right) \\
-H_{\perp}\left(M_{2} \cos \alpha_{1}+M_{3} \cos \left(\alpha_{1}+\alpha_{2}\right)\right) \\
-\kappa \alpha_{2}+H_{\|} M_{3} \sin \left(\alpha_{1}+\alpha_{2}\right)-H_{\perp} M_{3} \cos \left(\alpha_{1}+\alpha_{2}\right)
\end{array}\right)
$$

and $M$ is a matrix that depends only on $\alpha_{1}$ and $\alpha_{2}$. Its expression is given in the Appendix.

Remark 1: If the orthogonal magnetic field is equal to zero, (i.e. $H_{\perp}=0$ for all times), for any $H_{\|}$, states of the form $(x, y, \theta, 0,0)$ with $(x, y, \theta) \in \mathbf{R}^{3}$ are equilibrium positions. In particular, the parallel component of the magnetic field has no action on the swimmer when its shape is a straight line. This makes the system more difficult to control around the equilibrium. This issue is being dealt with in [11], where a modified swimmer model that is bent at equilibrium is introduced.

Remark 2: The problem is invariant by translation and rotation, as can be seen from the absence of $x$ and $y$ in the dynamics, and the special way in which the dynamics depends on $\theta$. Therefore, we focus on the equilibrium position $(0,0,0,0,0)$, without loss of generality.

Straightforward computations show that the determinant of $M$ remains negative for all values of $\alpha_{1}$ and $\alpha_{2}$, so $M$ is invertible and we can rewrite the system $(S)$ as a nonlinear control system given by

$$
\dot{Z}=\mathbf{F}_{0}+H_{\|} \mathbf{F}_{1}+H_{\perp} \mathbf{F}_{2},
$$

where $\mathbf{F}_{0}, \mathbf{F}_{1}$ and $\mathbf{F}_{2}$ are combinations of the third, fourth and fifth columns of $\left(M R_{-\theta}\right)^{-1}$, denoted respectively by $\mathbf{X}_{3}, \mathbf{X}_{4}$ and $\mathbf{X}_{5}$ :

$$
\begin{aligned}
\mathbf{F}_{0}= & -\kappa\left(\alpha_{1} \mathbf{X}_{4}+\alpha_{2} \mathbf{X}_{5}\right) \\
\mathbf{F}_{1}= & \left(M_{2} \sin \alpha_{1}+M_{3} \sin \left(\alpha_{1}+\alpha_{2}\right)\right)\left(\mathbf{X}_{3}+\mathbf{X}_{4}\right) \\
& +M_{3} \sin \left(\alpha_{1}+\alpha_{2}\right) \mathbf{X}_{5} \\
\mathbf{F}_{2}= & -M_{1} \mathbf{X}_{3} \\
& -\left(M_{2} \cos \alpha_{1}+M_{3} \cos \left(\alpha_{1}+\alpha_{2}\right)\right)\left(\mathbf{X}_{3}+\mathbf{X}_{4}\right) \\
& -M_{3} \cos \left(\alpha_{1}+\alpha_{2}\right) \mathbf{X}_{5}
\end{aligned}
$$

\section{LOCAL CONTROLLABILITY AROUND EQUILIBRIUM STATES}

\section{A. Small-time local controllability (STLC)}

We start with some useful definitions and properties. Let

$$
\dot{z}=f_{0}(z)+u_{1}(t) f_{1}(z)+u_{2}(t) f_{2}(z) .
$$

be a general nonlinear control-affine system, with $z$ in $\mathbf{R}^{n}$, $f_{0}, f_{1}, f_{2}$ real analytic vector fields in $\mathbf{R}^{n}$ and $u_{1}, u_{2}$ control 
functions in $L^{\infty}([0, T])$ for some $T>0$. For $\eta>0$, and $z \in \mathbf{R}^{n}$, let $B(z, \eta)$ be the open ball centered at $z$ with radius $\eta$. The following definition appears in [12, Definition 3.2].

Definition 1 (STLC): Let $z_{e}$ in $\mathbf{R}^{n}$, and $u_{e}=\left(u_{1 e}, u_{2 e}\right)$ constant controls such that $\left(z_{e}, u_{e}\right)$ is an equilibrium of the system (3). The control system (3) is STLC at $\left(z_{e}, u_{e}\right)$ if, for every $\varepsilon>0$, there exists $\zeta>0$ such that, for every $z_{0}, z_{1}$ in $B\left(z_{e}, \zeta\right)$, there exists controls $u_{1}(\cdot)$ and $u_{2}(\cdot)$ in $L^{\infty}([0, \varepsilon])$ such that the solution of the control system $z(\cdot):[0, \varepsilon] \rightarrow \mathbf{R}^{n}$ of (3) satisfies $z(0)=z_{0}, z(\varepsilon)=z_{1}$, and

$$
\left\|u_{1}-u_{1 e}\right\|_{L^{\infty}} \leqslant \varepsilon,\left\|u_{2}-u_{2 e}\right\|_{L^{\infty}} \leqslant \varepsilon .
$$

In the following, we assume that $f_{0}(0)=0$, such that $(0,(0,0))$ (state and controls equal to zero) is an equilibrium of the system 3 .

The following definitions and theorem provide a sufficient condition for STLC, that we will use later to prove our controllability result on the 3-link swimmer.

Definition 2 (LARC): System (3) satisfies the Lie Algebra Rank Condition (LARC) at 0 if the values at 0 of all iterated Lie brackets of the vector fields $f_{0}, f_{1}, f_{2}$ span a vector space of dimension $n$.

Let us introduce some notions of weight and orders of iterated Lie brackets (see [13, pp.184-185] for details). For $h$ an iterated Lie bracket involving the vector fields $f_{0}, f_{1}, f_{2}$, let $g$ the iterated Lie bracket obtained by exchanging $f_{1}$ and $f_{2}$ in $h$ (e.g., if $h=\left[f_{1},\left[f_{1},\left[f_{0}, f_{2}\right]\right]\right]$, then $\left.g=\left[f_{2},\left[f_{2},\left[f_{0}, f_{1}\right]\right]\right]\right)$. Let $\sigma(h)=h+g$, and let $\delta_{i}(h) \in \mathbf{N}(i=0,1,2)$ be the number of times $f_{i}$ appears in $h$, and $\rho(h)=\delta_{0}(h)+\delta_{1}(h)+\delta_{2}(h)$ (i.e. $\rho$ is the order of $h$ ). Let $G_{\rho(h)}$ be the subspace of $\mathbf{R}^{n}$ spanned by the value at 0 of all the iterated brackets $g$ such that $\rho(g)<\rho(h)$ (i.e. all the brackets of order inferior to the order of $h$ ).

Definition 3 (Sussmann's condition S): System (3) satisfies the condition $S$ at 0 if it satisfies the LARC and any iterated Lie bracket $h$ of the vector fields $f_{0}, f_{1}, f_{2}$ such that $\delta_{0}(h)$ is odd and $\delta_{1}(h)$ and $\delta_{2}(h)$ are even (those are called the "bad" brackets) satisfies $\sigma(h)(0) \in G_{\rho(h)}$.

Remark 3: Condition $S$ is called $S(1)$ in [13, Theorem 7.3], where a more general condition $S(\theta)$ is defined for $\theta \in[0,1]$.

Theorem 1 ([13], Theorem 7.3): If the Sussmann condition $S$ holds, system (3) is STLC.

\section{B. Controllability result for the 3-link swimmer}

In this section, we state a local controllability result for system $(S)$, around the equilibrium position $(0,0,0,0,0)$ (that we will denote by 0 for the sake of readability) with nonzero controls.

From now on, we assume that the physical constants $\ell, \eta$, $\xi, \kappa$ are positive, and that $\eta>\xi$. This is usually true in the swimmer's physical setting (for thin filaments, one typically has $\eta=2 \xi$, see [8]), and avoid dealing with numerous subcases.

Let $m=M_{1}+M_{3}$ and $\mu=M_{1}-M_{3}$. Before stating the result, we need to make a few technical assumptions about the magnetizations.
Assumption 1: The magnetizations $M_{1}, M_{2}$ and $M_{3}$ are such that

$$
\begin{array}{r}
\mu \neq 0 \\
\neq \\
\left(m \neq 0 \text { or } M_{2} \neq 0\right) \\
-7 M_{2}^{2}+9 M_{2} m-5 M_{1} M_{3} \neq 0 \\
P\left(M_{1}, M_{2}, M_{3}\right) \neq 0
\end{array}
$$

with

$$
\begin{aligned}
& P(x, y, z)=49 y^{3}-91 y^{2}(x+z) \\
& \quad+36 y(x+z)^{2}-(45 y+65(x+z)) x z .
\end{aligned}
$$

Remark 4: It is shown in [14, Section3.1], using a symmetry argument, that if $\mu=0$, the swimmer starting from the straight shape verifies $\alpha_{1}(t)=-\alpha_{2}(t)$ for all times. A similar argument shows that if $m=0$ and $M_{2}=0$, one has $\alpha_{1}(t)=\alpha_{2}(t)$ for all times. Therefore, the system is not controllable in both of these cases.

If $-7 M_{2}^{2}+9 M_{2} m-5 M_{1} M_{3}=0$, then the constant $\gamma$ expressed in Theorem 2 below is not defined. In that case, the swimmer is not STLC at $(0,(\beta, 0))$ for any $\beta \in \mathbf{R}$; see Remark 5 for further discussion about the uniqueness of $\gamma$.

The first three conditions in equation (4) are hence necessary for controllability. It is unclear whether the last one is also necessary. The polynomial expression $P$ seems to be of importance in the swimmer's dynamics, as it appears in all the determinants computed in the proof of Lemma 1 below. The values of $\left(M_{1}, M_{2}, M_{3}\right)$ for which $P$ vanishes may therefore correspond with cases where the swimmer's movement ability is limited. It seems nonetheless difficult to confirm this hypothesis analytically or numerically.

We now state our main result.

Theorem 2: System $(S)$ is STLC at $(0,(\gamma, 0))$ with

$$
\gamma=\kappa \frac{17 m-16 M_{2}}{-7 M_{2}^{2}+9 M_{2} m-5 M_{1} M_{3}} .
$$

Proof:

Let $T>0$. Let $H_{\|}$and $H_{\perp}$ be control functions defined on $[0, T]$. We define $\widetilde{H}_{\|}$as the affine feedback transformation $\widetilde{H}_{\|}=H_{\|}+\gamma$. With this new control, system $(S)$ can be written as a different control system

$$
\dot{Z}=\widetilde{\mathbf{F}}_{0}+\widetilde{H}_{\|} \widetilde{\mathbf{F}}_{1}+H_{\perp} \widetilde{\mathbf{F}}_{2},
$$

with $\widetilde{\mathbf{F}}_{0}=\mathbf{F}_{0}-\gamma \mathbf{F}_{1}, \widetilde{\mathbf{F}}_{1}=\mathbf{F}_{1}$ and $\widetilde{\mathbf{F}}_{2}=\mathbf{F}_{2}$.

For $i_{1}, \ldots, i_{m}$ indices in $\{0,1,2\}$, we denote by $F_{i_{1} i_{2} \ldots i_{m}}$ the value at 0 of the iterated Lie bracket $\left[\mathbf{F}_{i_{1}},\left[\mathbf{F}_{i_{2}}, \ldots \mathbf{F}_{i_{m}}\right] \ldots\right]$ of vector fields $\mathbf{F}_{0}, \mathbf{F}_{1}, \mathbf{F}_{2}$, and by $\widetilde{F}_{i_{1} i_{2} \ldots i_{m}}$ the value at 0 of the iterated Lie bracket $\left[\widetilde{\mathbf{F}}_{i_{1}},\left[\widetilde{\mathbf{F}}_{i_{2}}, \ldots \widetilde{\mathbf{F}}_{i_{m}}\right] \ldots\right]$ of vector fields $\widetilde{\mathbf{F}}_{0}, \widetilde{\mathbf{F}}_{1}, \widetilde{\mathbf{F}}_{2}$. For example, $F_{102}=\left[\mathbf{F}_{1},\left[\mathbf{F}_{0}, \mathbf{F}_{2}\right]\right](0)$ and $\widetilde{F}_{102}=\left[\widetilde{\mathbf{F}}_{1},\left[\widetilde{\mathbf{F}}_{0}, \widetilde{\mathbf{F}}_{2}\right]\right](0)$.

We start with two lemmas on the Lie brackets of the systems $(S)$ and $(\tilde{S})$.

Lemma 1: One has

$$
\widetilde{F}_{101}=0, \quad \widetilde{F}_{202}=0,
$$

and

$$
\operatorname{Span}\left\{\widetilde{F}_{2}, \widetilde{F}_{02}, \widetilde{F}_{12}, \widetilde{F}_{212}, \widetilde{F}_{2202}, \widetilde{F}_{2212}\right\}=\mathbf{R}^{5}
$$


Proof: Using a computer algebra software (for example Mathematica), we compute Lie brackets and show (5). We also show that only the first component of $\widetilde{F}_{212}$ is nonzero, and its value is equal to

$$
48 \frac{(\xi-\eta) \mu}{5 \ell^{8} \xi^{3} \eta}\left(-7 M_{2}^{2}+9 M_{2} m-5 M_{1} M_{3}\right),
$$

that is nonzero thanks to Assumption 1. Moreover, the determinants of $\left(\widetilde{F}_{2}, \widetilde{F}_{02}, \widetilde{F}_{12}, \widetilde{F}_{212}, \widetilde{F}_{2202}\right)$ and $\left(\widetilde{F}_{2}, \widetilde{F}_{02}, \widetilde{F}_{12}, \widetilde{F}_{212}, \widetilde{F}_{2212}\right)$ cannot be both zero if Assumption 1 holds. Hence (6) is verified.

Lemma 2: One has

$$
F_{101}=0, \quad F_{202}=\gamma F_{212},
$$

and

$$
\operatorname{Span}\left\{F_{2}, F_{02}, F_{12}, F_{212}, F_{2202}, F_{2212}\right\}=\mathbf{R}^{5} .
$$

Proof: Since $\mathbf{F}_{0}=\widetilde{\mathbf{F}}_{0}+\gamma \mathbf{F}_{1}, \mathbf{F}_{1}=\widetilde{\mathbf{F}}_{1}$ and $\mathbf{F}_{2}=\widetilde{\mathbf{F}}_{2}$, one immediately has (7) and (8).

Lemmas 1 and 2 allow to prove the following results:

Proposition 1: System $(S)$ does not satisfy the Sussmann condition $S$.

Theorem 3: System $(\tilde{S})$ is STLC at $(0,(0,0))$.

Proof: We prove simultaneously Proposition 1 and Theorem 3. System $(S)$ (resp. system $(\tilde{S})$ ) satisfies the LARC thanks to (8) (resp. (6)). Moreover, all the "bad" brackets $h$ such that $\rho(h)>4$ trivially belong to $G_{\rho(h)}=\mathbf{R}^{5}$. The only bad brackets of lower order that remain are the ones with two times $\mathbf{F}_{1}$ or $\mathbf{F}_{2}$ and one time $\mathbf{F}_{0}$ (resp. $\widetilde{\mathbf{F}}_{0}$ ). The Sussmann condition $S$ requires $F_{101}+F_{202}$ (resp. $\widetilde{F}_{101}+\widetilde{F}_{202}$ ) to belong to the subspace spanned by the brackets of order smaller than 2 , which is $\operatorname{Span}\left\{F_{2}, F_{02}, F_{12}\right\}$ (resp. $\operatorname{Span}\left\{\widetilde{F}_{2}, \widetilde{F}_{02}, \widetilde{F}_{12}\right\}$ ).

Equations (7) and (8) show that $F_{101}+F_{202}=\gamma F_{212} \notin$ $\operatorname{Span}\left\{F_{2}, F_{02}, F_{12}\right\}$, which proves Proposition 1. On the other hand, (5) shows that $\widetilde{F}_{101}+\widetilde{F}_{202}=0 \in \operatorname{Span}\left\{\widetilde{F}_{2}, \widetilde{F}_{02}, \widetilde{F}_{12}\right\}$, which proves that the condition $S$ is true for $(\tilde{S})$. Then, thanks to Theorem $1,(\tilde{S})$ is STLC at $(0,(0,0))$.

We now conclude the proof of Theorem 2, as a corollary to Theorem 3. Let $\varepsilon>0$. Let $\zeta$ be the associated parameter from Definition 1 , and $Z_{0}, Z_{1}$ in $B(0, \zeta)$. There exists controls $\widetilde{H}_{\|}$ and $H_{\perp}$ defined on $[0, \varepsilon]$ such that the solution of $(\tilde{S})$ with $Z(0)=Z_{0}$ and these controls verifies $Z(\varepsilon)=Z_{1}$, and

$$
\left\|\widetilde{H}_{\|}\right\|_{L^{\infty}} \leqslant \varepsilon,\left\|H_{\perp}\right\|_{L^{\infty}} \leqslant \varepsilon .
$$

Hence, the solution of system $(S)$ with $Z(0)=Z_{0}$ and controls $H_{\|}=\widetilde{H}_{\|}-\gamma$ and $H_{\perp}$ verifies $Z(\varepsilon)=Z_{1}$. Moreover, $\left\|H_{\|}-\gamma\right\|_{L^{\infty}} \leqslant \varepsilon$ and $\left\|H_{\perp}\right\|_{L^{\infty}} \leqslant \varepsilon$.

Remark 5: In a forthcoming paper [15], we show that $\gamma$ is unique: system $(S)$ is not STLC around any control different from $(\gamma, 0)$. In particular, one cannot hope to control the system at $(0,(0,0))$ (i.e. with small controls) or at $(0,(\delta, 0))$ with $\delta<\gamma$. Our result is optimal in that sense.

The uniqueness of $\gamma$ is due to the fact that it is the only value that allows to "neutralize" the bracket $F_{202}$ with the bracket $F_{212}$ at 0 . Around another control $(0,(\delta, 0))$ with $\delta \neq \gamma$, one has to check that $F_{202}$ obstructs local controllability and that all the other brackets cannot "neutralize" it. This requires a careful study of the higher-order brackets. The calculations in their full length would exceed the scope of the present study. The reader is invited to refer to [15] for more details.

\section{A similar result for the 2-link swimmer}

In [9], the authors conduct a study on the 2-link magnetic swimmer and state a local controllability result. However, the result they state is weaker than STLC. In this section, we improve this result, using the same arguments than for the 3-link swimmer.

The notations used in the following are the same as in their paper, and as above: each of the two segments has length $\ell$, hydrodynamic coefficients $\eta$ and $\xi$; the segments are magnetized with magnetizations $M_{1}$ and $M_{2}$ and connected by a torsional spring with stiffness $\kappa$. The swimmer is submitted to a magnetic field $\left(H_{\|}, H_{\perp}\right)$. The state variables are $x, y, \theta$, defined as for the 3-link swimmer, and $\alpha$, the angle between the two links. We assume that $\ell, \eta, \xi$ and $\kappa$ are positive and that $\eta>\xi$.

The derivation of the dynamics equation leads to a system analog to system $(S)$

$$
\dot{Y}=\mathbf{G}_{0}+H_{\|}(t) \mathbf{G}_{1}+H_{\perp}(t) \mathbf{G}_{2},
$$

with $Y=(x, y, \theta, \alpha)$.

Without loss of generality (thanks to an argument similar to Remark 2), we focus on controllability around the position 0 (i.e. $(0,0,0,0)$ ) with nonzero controls. Using the "return method" from [12, Chapter 6], the following result is shown in [9, Theorem III.4]:

Theorem 4: Assume $M_{1} M_{2} \neq 0$ and $M_{1} \neq M_{2}$. Let $\varepsilon>0$. For any $Y_{0}, Y_{1}$ in $B(0, \zeta)$, there exist $H_{\|}$and $H_{\perp}$ in $L^{\infty}[0, \epsilon]$ such that the solution of $(\Sigma)$ with $Y(0)=Y_{0}$ and these controls verifies $Y(\varepsilon)=Y_{1}$, and

$$
\left\|H_{\|}\right\|_{L^{\infty}} \leqslant 2 \kappa\left|\frac{1}{M_{1}}+\frac{1}{M_{2}}\right|+\varepsilon, \quad\left\|H_{\perp}\right\|_{L^{\infty}} \leqslant \varepsilon .
$$

Before stating our result, let us point out a few particular cases, as for the 3-link swimmer.

Proposition 2: If $M_{1}=0$ or $M_{2}=0$, the swimmer is not STLC.

Proof: We start with a useful lemma.

Lemma 3: If $\mathbf{G}_{1}=0$, and $G_{202} \notin$ $\operatorname{Span}\left\{G_{2}, G_{02}, \ldots, G_{0 . \ldots 02}, \ldots\right\}$, then System $(\Sigma)$ is not STLC.

Proof: This is a direct application of the necessary condition stated in [16, Prop. 6.3, p.707], for scalar-input control systems.

The symmetry of the system makes both cases $M_{1}=0$ and $M_{2}=0$ equivalent. Moreover, in the case $M_{1}=0$, a straightforward computation shows $\mathbf{G}_{1}=0$ and $G_{202} \notin$ $\operatorname{Span}\left\{G_{2}, G_{02}, \ldots, G_{0 \ldots 02}, \ldots\right\}$, so we can apply Lemma 3 .

From now on, we make the following assumptions on the magnetizations:

Assumption 2: The magnetizations $M_{1}$, and $M_{2}$ are such that $M_{1} \neq 0, M_{2} \neq 0, M_{1}-M_{2} \neq 0$ and $M_{1}+M_{2} \neq 0$. 
Remark 6: It is shown in [9, Section III] that the system is not controllable if $M_{1}-M_{2}=0$, and STLC at $(0,(0,0))$ if $M_{1}+M_{2}=0$. Moreover, it is shown in [17] that, unless $M_{1}+M_{2}=0$, the 2-link swimmer is not STLC at $(0,(0,0))$.

We now state our result for the 2-link swimmer.

Theorem 5: System $(\Sigma)$ is STLC at $\left(0,\left(\gamma^{\prime}, 0\right)\right)$ with

$$
\gamma^{\prime}=\kappa\left(\frac{1}{M_{1}}+\frac{1}{M_{2}}\right) \text {. }
$$

Remark 7: This improves the result from Theorem 4, for it shows that the system is STLC at $\left(\gamma^{\prime}, 0\right)$, whereas Theorem 4 does not require the control $H_{\|}$to stay arbitrarily close to the upper bound $2 \kappa\left|\frac{1}{M_{1}}+\frac{1}{M_{2}}\right|$. This upper bound on $H_{\|}$is also improved in our result.

Proof: Let $T>0$. Let $H_{\|}$and $H_{\perp}$ be control functions defined on $[0, T]$. Let

We define $\widetilde{H}_{\|}=H_{\|}+\gamma^{\prime}$ as above, to get the feedback system

$$
\dot{Y}=\widetilde{\mathbf{G}}_{0}+\widetilde{H}_{\|} \widetilde{\mathbf{G}}_{1}+H_{\perp} \widetilde{\mathbf{G}}_{2},
$$

with $\widetilde{\mathbf{G}}_{0}=\mathbf{G}_{0}-\gamma^{\prime} \mathbf{G}_{1}, \widetilde{\mathbf{G}}_{1}=\mathbf{G}_{1}$ and $\widetilde{\mathbf{G}}_{2}=\mathbf{G}_{2}$.

We will use the same notations as above for the Lie brackets associated to systems $(\Sigma)$ and $(\tilde{\Sigma})$, evaluated at 0 . For example, $G_{102}=\left[\mathbf{G}_{1},\left[\mathbf{G}_{0}, \mathbf{G}_{2}\right]\right](0)$ and $\widetilde{G}_{102}=\left[\widetilde{\mathbf{G}}_{1},\left[\widetilde{\mathbf{G}}_{0}, \widetilde{\mathbf{G}}_{2}\right]\right](0)$.

Lemma 4: One has $\operatorname{Span}\left\{G_{2}, G_{12}, G_{212}, G_{2202}\right\}=\mathbf{R}^{4}$ and

$$
G_{101}=0, \quad G_{202}=\gamma^{\prime} G_{212} .
$$

Proof: Using a computer algebra software, we compute Lie brackets and show (9). We also show that the first component of each of the three vectors $G_{2}, G_{12}, G_{2202}$ is zero. The determinant $D$ of the matrix formed with the three last components of these vectors expresses

$$
D=\frac{209952 \kappa M_{1} M_{2}(\eta-\xi)\left(M_{1}^{2}-M_{2}^{2}\right)^{2}}{\eta^{7} l^{20} \xi} .
$$

$D$ is nonzero thanks to Assumption 2. Moreover, only the first component of $G_{212}$ is nonzero, and its value is equal to

$$
\frac{216 M_{1} M_{2}(\eta-\xi)\left(M_{1}-M_{2}\right)}{\eta^{3} l^{8} \xi}
$$

that is nonzero thanks to Assumption 2. Hence (8) is verified.

Lemma 5: One has $\operatorname{Span}\left\{\widetilde{G}_{2}, \widetilde{G}_{12}, \widetilde{G}_{212}, \widetilde{G}_{2202}\right\}=\mathbf{R}^{4}$ and

$$
\widetilde{G}_{101}=0, \quad \widetilde{G}_{202}=\gamma^{\prime} \widetilde{G}_{212}
$$

Proof: Since $\widetilde{\mathbf{G}}_{0}=\mathbf{G}_{0}-\gamma \mathbf{G}_{1}, \widetilde{\mathbf{G}}_{1}=\mathbf{G}_{1}$ and $\widetilde{\mathbf{G}}_{2}=\mathbf{G}_{2}$, one immediately has (10).

Proposition 3: System $(\Sigma)$ does not satisfy the Sussmann condition $S$.

Remark 8: This proposition is stated and shown in [9, Prop. III.11].

Theorem 6: System $(\tilde{\Sigma})$ is STLC at $(0,(0,0))$.

Proof: See the proofs of Proposition 1 and Theorem 3 in the previous section.

We conclude the proof of Theorem 5 as in the previous section.

\section{Discussion}

\section{A. Comments on the main results}

Theorem 2 is, to our knowledge, the first local controllability result for this magnetically actuated 3-link swimmer. It shows, rather counterintuitively, that the parallel component of the magnetic field needs to remain large in order to control the swimmer, even when the target state is very close to its equilibrium position. In the particular case $16 m-17 M_{2}=0$, the constant $\gamma$ is equal to 0 , and the standard STLC at $(0,(0,0))$ is retrieved. We improved the existing result on the 2-link swimmer in Theorem 5.

Physically, these results reflect the fact that the parallel component of the magnetic field has no effect on the swimmer when it is at its equilibrium shape, i.e. when all the segments are aligned. This may be seen as a loss of controllability at the equilibrium. The parallel control $H_{\|}$plays however a crucial role in the controllability properties of the swimmers.

This result provides a useful insight for experiments, by showing that the 2- and 3-link swimmers may not be driven easily in any direction from an equilibrium point, and giving an explicit lower bound on the control needed to achieve local controllability. Further work on the subject of microswimmers, currently under our investigation, is to consider swimmers with more links, that describe more realistically flexible filaments. This work also addresses the question of the existence of necessary conditions for local controllability for systems with non-scalar controls, for which little is known.

\section{B. Numerical simulations}

In order to numerically observe the local behavior of the system, we steer it from an equilibrium state with different controls that stay "close" to the equilibrium control. Let $\beta$ be a real number and $\epsilon>0$ be a small parameter; we set

$$
\begin{aligned}
& H_{\|}(t)=\beta+\varepsilon\left(h_{1}+h_{2} \cos (10 t)+h_{3} \cos (100 t)\right) \\
& H_{\perp}(t)=\varepsilon\left(h_{4}+h_{5} \cos (10 t)+h_{3} \cos (100 t)\right)
\end{aligned}
$$

with $h_{1}$ to $h_{6}$ constants taken randomly in $[-1,1]$. We take $N$ realizations of these random controls and solve the 2-link swimmer system starting from $(0,0,0,0)$ over the time interval $[0, T]$. With such a range of randomized oscillating controls close to $(\beta, 0)$, we expect the obtained trajectories to roughly cover the reachable space in small time $T$, which allows to observe the unattainable regions if there are any. The results of the simulations, performed with MATLAB, for the 2-link swimmer with these controls are displayed on Figure 2. When $\beta$ is different from the critical value $\gamma^{\prime}$, the trajectories remain, locally, either always left or always right of $(0,0)$ in the $2 d-$ plane, which tends to validate the non-STLC of the swimmer. On the contrary, for $\beta=\gamma^{\prime}$, where the swimmer can be locally controlled according to our result, the trajectories seem to cover a neighborhood of 0 .

The dynamics of the 3-link swimmer are more complex and appear to be numerically unstable. The oscillating controls generate numerical artifacts over the trajectories; hence the numerical simulations in this case are less conclusive than for the two-link swimmer. 


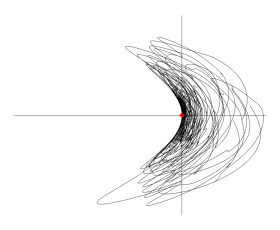

(a) $\beta=0.5 \gamma^{\prime}$

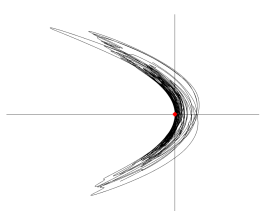

(b) $\beta=0.9 \gamma^{\prime}$

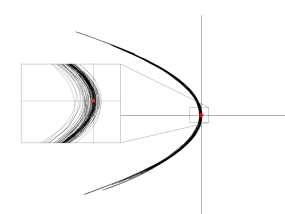

(c) $\beta=\gamma^{\prime}$

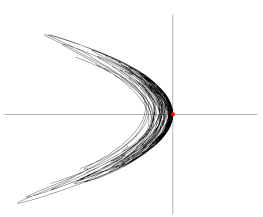

(d) $\beta=1.1 \gamma^{\prime}$

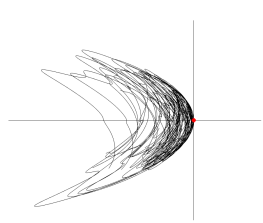

(e) $\beta=1.25 \gamma^{\prime}$

Fig. 2. Illustration of the role played by the constant $\gamma^{\prime}$. On each graph are plotted $N=30$ trajectories in the $2 \mathrm{~d}$-plane of the extremity of the 2-link swimmer, starting at state $(0,0,0,0)$ and with the randomized controls $(11)$ taken "around" $(\beta, 0)$, for 5 different values of $\beta$. The origin $(0,0)$ is indicated by the red dot on each graph. One can observe that a certain region seems to be unattainable for every case, except for the case $\beta=\gamma^{\prime}$ (graph (c)) where controllability at $(0,0)$ is theoretically retrieved (a zoom-in around the origin is added on the graph to show that the trajectories cover a neighborhood of $(0,0)$. The numerical values used are $\eta=4, \xi=2, l=1, M_{1}=1, M_{2}=3, k=1, \varepsilon=10^{-2}$, and $T=1$.

\section{APPENDIX}

We provide the detailed expression of the matrix $M\left(\alpha_{1}, \alpha_{2}\right)$ introduced in (2). $M$ is of size $5 \times 5$ and its entries read

$$
\begin{aligned}
& m_{11}=\ell(\eta+2 \xi) \sin \left(\alpha_{1}\right) \sin \left(\alpha_{2}\right)-3 l \xi \cos \left(\alpha_{1}\right) \cos \left(\alpha_{2}\right), \\
& m_{12}=-\ell\left((\eta+2 \xi) \cos \left(\alpha_{2}\right) \sin \left(\alpha_{1}\right)+(2 \eta+\xi) \cos \left(\alpha_{1}\right) \sin \left(\alpha_{2}\right)\right) \text {, } \\
& m_{13}=-\frac{1}{2} \ell^{2}\left((\eta+4 \xi) \cos \left(\alpha_{2}\right) \sin \left(\alpha_{1}\right)\right. \\
& \left.+\left(\eta+2 \xi+(3 \eta+2 \xi) \cos \left(\alpha_{1}\right)\right) \sin \left(\alpha_{2}\right)\right) \text {, } \\
& m_{14}=-\frac{1}{2} \ell^{2}(\eta+2 \xi) \sin \left(\alpha_{2}\right) \text {, } \\
& m_{15}=0 \text {, } \\
& m_{21}=\ell(2 \eta+\xi) \cos \left(\alpha_{2}\right) \sin \left(\alpha_{1}\right)+l(\eta+2 \xi) \cos \left(\alpha_{1}\right) \sin \left(\alpha_{2}\right), \\
& m_{22}=\ell(2 \eta+\xi) \sin \left(\alpha_{1}\right) \sin \left(\alpha_{2}\right)-3 l \eta \cos \left(\alpha_{1}\right) \cos \left(\alpha_{2}\right) \text {, } \\
& m_{23}=-\frac{1}{2} \ell^{2}\left(\left(5 \cos \left(\alpha_{1}\right)+3\right) \cos \left(\alpha_{2}\right) \eta+\eta-(3 \eta+2 \xi) \sin \left(\alpha_{1}\right) \sin \left(\alpha_{2}\right)\right) \text {, } \\
& m_{24}=-\frac{1}{2} \ell^{2} \eta\left(3 \cos \left(\alpha_{2}\right)+1\right) \\
& m_{25}=-\frac{1}{2}\left(\ell^{2} \eta\right) \text {, } \\
& m_{31}=\frac{1}{2} \ell^{2}\left((2 \eta+\xi) \sin \left(\alpha_{1}\right)+(\eta-\xi) \sin \left(2 \alpha_{1}\right)+\eta \sin \left(\alpha_{1}+\alpha_{2}\right)\right. \\
& \left.+(\eta-\xi)\left(\sin \left(2\left(\alpha_{1}+\alpha_{2}\right)\right)+\sin \left(\alpha_{1}+2 \alpha_{2}\right)\right)\right) \text {, } \\
& m_{32}=-\frac{1}{2} \ell^{2}\left(\cos \left(\alpha_{1}+\alpha_{2}\right) \eta+\cos \left(2\left(\alpha_{1}+\alpha_{2}\right)\right) \eta+3 \eta+2 \xi\right. \\
& +(2 \eta+\xi) \cos \left(\alpha_{1}\right)+(\eta-\xi) \cos \left(2 \alpha_{1}\right) \\
& \left.-\xi \cos \left(2\left(\alpha_{1}+\alpha_{2}\right)\right)+(\eta-\xi) \cos \left(\alpha_{1}+2 \alpha_{2}\right)\right) \text {, } \\
& m_{33}=-\frac{1}{2} \ell^{3}\left(2 \cos \left(\alpha_{2}\right) \eta+\cos \left(2 \alpha_{2}\right) \eta+2 \cos \left(\alpha_{1}+\alpha_{2}\right) \eta\right. \\
& +\cos \left(2\left(\alpha_{1}+\alpha_{2}\right)\right) \eta+5 \eta+3 \xi+2(2 \eta+\xi) \cos \left(\alpha_{1}\right) \\
& +(\eta-\xi) \cos \left(2 \alpha_{1}\right)-\xi \cos \left(2 \alpha_{2}\right)-\xi \cos \left(2\left(\alpha_{1}+\alpha_{2}\right)\right) \\
& \left.+2(\eta-\xi) \cos \left(\alpha_{1}+2 \alpha_{2}\right)\right) \text {, } \\
& m_{34}=-\frac{1}{6} \ell^{3}\left(7 \eta+3 \xi+3\left((2 \eta+\xi) \cos \left(\alpha_{1}\right)+2 \eta \cos \left(\alpha_{2}\right)+\eta \cos \left(2 \alpha_{2}\right)\right.\right. \\
& \left.\left.-\xi \cos \left(2 \alpha_{2}\right)+\eta \cos \left(\alpha_{1}+\alpha_{2}\right)+(\eta-\xi) \cos \left(\alpha_{1}+2 \alpha_{2}\right)\right)\right) \text {, } \\
& m_{35}=-\frac{1}{6} \ell^{3} \eta\left(3 \cos \left(\alpha_{2}\right)+3 \cos \left(\alpha_{1}+\alpha_{2}\right)+2\right) \text {, } \\
& m_{41}=\frac{1}{2} \ell^{2}\left((2 \eta+\xi) \sin \left(\alpha_{1}\right)+\eta \sin \left(\alpha_{1}+\alpha_{2}\right)+(\eta-\xi) \sin \left(\alpha_{1}+2 \alpha_{2}\right)\right), \\
& m_{42}=-\frac{1}{2} \ell^{2}\left((2 \eta+\xi) \cos \left(\alpha_{1}\right)+\eta \cos \left(\alpha_{1}+\alpha_{2}\right)+(\eta-\xi) \cos \left(\alpha_{1}+2 \alpha_{2}\right)\right) \text {, } \\
& m_{43}=-\frac{1}{6} \ell^{3}\left(7 \eta+3 \xi+3\left((2 \eta+\xi) \cos \left(\alpha_{1}\right)+2 \eta \cos \left(\alpha_{2}\right)+\eta \cos \left(2 \alpha_{2}\right)\right.\right. \\
& \left.\left.-\xi \cos \left(2 \alpha_{2}\right)+\eta \cos \left(\alpha_{1}+\alpha_{2}\right)+(\eta-\xi) \cos \left(\alpha_{1}+2 \alpha_{2}\right)\right)\right) \\
& m_{44}=-\frac{1}{6} \ell^{3}\left(6 \cos \left(\alpha_{2}\right) \eta+7 \eta+3 \xi+3(\eta-\xi) \cos \left(2 \alpha_{2}\right)\right) \text {, } \\
& m_{45}=-\frac{1}{6} \ell^{3} \eta\left(3 \cos \left(\alpha_{2}\right)+2\right) \\
& m_{51}=\frac{1}{2} \ell^{2} \eta \sin \left(\alpha_{1}+\alpha_{2}\right), \\
& m_{52}=-\frac{1}{2} \ell^{2} \eta \cos \left(\alpha_{1}+\alpha_{2}\right) \text {, } \\
& m_{53}=-\frac{1}{6} \ell^{3} \eta\left(3 \cos \left(\alpha_{2}\right)+3 \cos \left(\alpha_{1}+\alpha_{2}\right)+2\right), \\
& m_{54}=-\frac{1}{6} \ell^{3} \eta\left(3 \cos \left(\alpha_{2}\right)+2\right), \\
& m_{55}=-\frac{1}{3} \ell^{3} \eta \text {, }
\end{aligned}
$$

\section{ACKNOWLEDGMENT}

The author thanks Laetitia Giraldi, from Inria, Pierre Lissy, from Université Paris-Dauphine, and Jean-Baptiste Pomet, from Inria, for their insight and advice that greatly assisted the research, and for their proofreading that helped improve the manuscript.

\section{REFERENCES}

[1] K. E. Peyer, L. Zhang, and B. J. Nelson, "Bio-inspired magnetic swimming microrobots for biomedical applications," Nanoscale, vol. 5, no. 4, pp. 1259-1272, 2013.

[2] W. Gao, D. Kagan, O. S. Pak, C. Clawson, S. Campuzano, E. ChuluunErdene, E. Shipton, E. E. Fullerton, L. Zhang, E. Lauga, et al., "Cargotowing fuel-free magnetic nanoswimmers for targeted drug delivery," small, vol. 8, no. 3, pp. 460-467, 2012.

[3] R. Dreyfus, J. Baudry, M. L. Roper, M. Fermigier, H. A. Stone, and J. Bibette, "Microscopic artificial swimmers," Nature, vol. 437, no. 7060, p. 862, 2005.

[4] A. Ghosh and P. Fischer, "Controlled propulsion of artificial magnetic nanostructured propellers," Nano letters, vol. 9, no. 6, pp. 2243-2245, 2009.

[5] E. Gutman and Y. Or, "Simple model of a planar undulating magnetic microswimmer," Physical Review E, vol. 90, no. 1, p. 013012, 2014.

[6] F. Alouges, A. DeSimone, L. Giraldi, and M. Zoppello, "Self-propulsion of slender micro-swimmers by curvature control: N-link swimmers," International Journal of Non-Linear Mechanics, vol. 56, pp. 132-141, 2013.

[7] E. M. Purcell, "Life at low reynolds number," American journal of physics, vol. 45, no. 1, pp. 3-11, 1977.

[8] J. Gray and G. Hancock, "The propulsion of sea-urchin spermatozoa," Journal of Experimental Biology, vol. 32, no. 4, pp. 802-814, 1955.

[9] L. Giraldi and J.-B. Pomet, "Local controllability of the two-link magneto-elastic micro-swimmer," IEEE Transactions on Automatic Control, vol. 62, no. 5, pp. 2512-2518, 2017.

[10] F. Alouges, A. DeSimone, L. Giraldi, and M. Zoppello, "Can magnetic multilayers propel artificial microswimmers mimicking sperm cells?" Soft Robotics, vol. 2, no. 3, pp. 117-128, 2015.

[11] L. Giraldi, P. Lissy, C. Moreau, and J.-B. Pomet, "Controllability of a bent 3-link magnetic microswimmer," arXiv preprint arXiv:1611.00993, 2016.

[12] J.-M. Coron, Control and nonlinearity, ser. Mathematical Surveys and Monographs. AMS, Providence, RI, 2007, vol. 136.

[13] H. J. Sussmann, "A general theorem on local controllability," SIAM Journal on Control and Optimization, vol. 25, no. 1, pp. 158-194, 1987.

[14] F. Alouges, A. Desimone, L. Giraldi, and M. Zoppello, "Purcell magneto-elastic swimmer controlled by an external magnetic field," IFAC-PapersOnLine, vol. 50, no. 1, pp. 4120-4125, 2017.

[15] L. Giraldi, P. Lissy, C. Moreau, and J.-B. Pomet, "A necessary condition for local controllability of a particular class of systems with two scalar controls," in preparation, 2019.

[16] H. J. Sussmann, "Lie brackets and local controllability: a sufficient condition for scalar-input systems," SIAM Journal on Control and Optimization, vol. 21, no. 5, pp. 686-713, 1983.

[17] L. Giraldi, P. Lissy, C. Moreau, and J.-B. Pomet, "Addendum to "Local controllability of the two-link magneto-elastic microswimmer"," IEEE Transactions on Automatic Control, vol. 63, pp. 2303-2305, 2018. 